\title{
Coherent phonon transport in short-period two-dimensional superlattices of graphene and boron nitride
}

\author{
Carlos da Silva, ${ }^{1}$ Fernan Saiz, ${ }^{2}$ David A. Romero, ${ }^{1}$ and Cristina H. Amon ${ }^{1, *}$ \\ ${ }^{1}$ Department of Mechanical and Industrial Engineering, University of Toronto, Toronto, Ontario, Canada M5S $3 G 8$ \\ ${ }^{2}$ Department of Chemistry, Imperial College, London SWZ 2A7, United Kingdom
}

(Received 29 November 2015; revised manuscript received 11 February 2016; published 22 March 2016)

\begin{abstract}
Promoting coherent transport of phonons at material interfaces is a promising strategy for controlling thermal transport in nanostructures and an alternative to traditional methods based on structural defects. Coherent transport is particularly relevant in short-period heterostructures with smooth interfaces and long-wavelength heat-carrying phonons, such as two-dimensional superlattices of graphene and boron nitride. In this work, we predict phonon properties and thermal conductivities in these superlattices using a normal mode decomposition approach. We study the variation of the frequency dependence of these properties with the periodicity and interface configuration (zigzag and armchair) for superlattices with period lengths within the coherent regime. Our results showed that the thermal conductivity decreases significantly from the first period length $(0.44 \mathrm{~nm})$ to the second period length $(0.87 \mathrm{~nm}), 13 \%$ across the interfaces and $16 \%$ along the interfaces. For greater periods, the conductivity across the interfaces continues decreasing at a smaller rate of $11 \mathrm{~W} / \mathrm{mK}$ per period length increase $(0.43 \mathrm{~nm})$, driven by changes in the phonon group velocities (coherent effects). In contrast, the conductivity along the interfaces slightly recovers at a rate of $2 \mathrm{~W} / \mathrm{mK}$ per period, driven by changes in the phonon relaxation times (diffusive effects). By changing the interface configuration from armchair to zigzag, the conductivities for all period lengths increase by approximately $7 \%$ across the interfaces and $19 \%$ along the interfaces.
\end{abstract}

DOI: 10.1103/PhysRevB.93.125427

\section{INTRODUCTION}

Miniaturization is no longer the only option pursued to improve the performance of electronic nanodevices. An alternative option is to engineer nanostructures such as nanowires [1], nanoribbons [2], and superlattices [3,4] using promising two-dimensional (2D) materials such as graphene [5], boron nitride [6], and molybdenum disulfide [7]. These 2D materials usually exhibit thermal conductivities significantly higher than those of bulk or thin-film samples of the same material [8]. Graphene, with a measured thermal conductivity as high as $1813 \mathrm{~W} / \mathrm{mK}$ [5], has probably received the most attention, but this material is unsuitable as the sole building block of transistors because it lacks an electronic band gap. In contrast, atomic layers of molybdenum disulfide and boron nitride typically exhibit a band gap and unique thermal properties, placing them at the forefront of the next-generation electronics [9]. The physical properties of layered materials have been engineered by applying a variety of techniques such as strain [10], doping [11], lattice defects [12], and electric fields [13]. In addition, superlattices made of alternating layers of these nanosheets have been proven to be effective ways to control the electronic and thermal properties. These layers can be vertically stacked to form three-dimensional (3D) superlattices [14] or placed periodically in plane to form 2D superlattices [15]. For example, the electronic band structure of 2D superlattices of graphene and boron nitride can be tuned in such a way that the hybrid nanomaterial undergoes a transition from a semiconductor at the shortest period length to a metal at higher period lengths [16]. However, little is known about the phonon thermal transport in these short-period superlattices.

\footnotetext{
*cristina.amon@utoronto.ca
}

The primitive lattice vectors of the honeycomb lattices of graphene and boron nitride are nearly the same, enabling the synthesis of superlattices with smooth interfaces [15]. These interfaces favor the specular scattering of phonons [17], making this combination of materials an excellent test scenario to evaluate the isolated effect of the period on the phonon transport in 2D superlattices. From a thermal point of view, superlattices have been mainly investigated for thermoelectric applications, where a minimum thermal conductivity is preferred. As the superlattice period increases, the thermal conductivity first decreases until it reaches a minimum value, and later it increases until diffusive effects dominate the thermal transport [17]. This minimum, representing the crossover between coherent (wavelike) and incoherent (particlelike) phonon transport, has been verified experimentally in 3D superlattices [18], and predicted theoretically in 2D superlattices [19]. In the coherent regime, where wave interference effects dominate the thermal transport [17], the thermal conductivity of 3D superlattices of silicon and germanium has been shown to surpass the thermal conductivities of its constituent materials if the period is the shortest possible [20], broadening the range of potential applications for these nanostructures. A rigorous phonon thermal transport analysis at this short-period limit is needed to verify this thermal behavior in 2D superlattices.

In this paper we investigate the interplay between phonon group velocities and phonon relaxation times, and the role of acoustic phonon modes in the thermal transport in shortperiod 2D superlattices of graphene and boron nitride. We estimate thermal conductivities and phonon properties in the directions across and along the interfaces, for superlattices with zigzag and armchair interfaces. Special emphasis is given to the variation of these properties with superlattice periods within the coherent phonon transport regime, starting at the short-period limit. This knowledge is key to developing the 
capability of designing superlattices to improve the thermal performance of 2D materials. The phonon group velocities, phonon frequencies, and eigendisplacements are estimated using harmonic lattice dynamics (HLD) simulations. We use density functional theory (DFT) simulations to relax the unit cells of the hybrid structures, and density functional perturbation theory (DFPT) to validate the empirically approximated phonon dispersion curves. The phonon relaxation times are extracted from molecular dynamics (MD) simulations by collecting atomic velocities that are later postprocessed using a normal mode decomposition (NMD) approach. Finally, we use the properties of individual phonon modes to estimate the thermal conductivity with a mode-dependent equation from kinetic theory. After this introduction, the rest of this paper is organized as follows: Section II describes the unit cells, the simulation domains, and the methods to compute the phonon properties and thermal conductivities. Section III presents the predicted phonon dispersion curves, group velocities, relaxation times, and thermal conductivities. Special emphasis is given to the role of the acoustic modes and the sensitivity of these properties to the superlattice period and interface configuration. Finally, Sec. IV summarizes the main findings.

\section{SIMULATION METHODS}

In this section, we describe the methodologies to assemble the 2D superlattices of graphene and boron nitride and to calculate their phonon properties and thermal conductivities. The unit cells of the superlattices with the shortest period, denoted as $1 \times 1$ zigzag [Fig. 1(a)] and $1 \times 1$ armchair [Fig. 1(b)], are formed by one irreducible block of boron nitride and one irreducible block of graphene. We have investigated superlattices with both interfaces and periods $1 \times 1,2 \times 2$, $3 \times 3,4 \times 4,5 \times 5,7 \times 7$, and $10 \times 10$. These unit cells are initially assembled with a uniform bond length of $0.143 \mathrm{~nm}$, which is the average value of those for the honeycomb lattices of boron nitride $(0.145 \mathrm{~nm})$ and graphene $(0.141 \mathrm{~nm})$ [21]. The equilibrium lattice parameters of these cells are then obtained via variable-cell optimization with the $a b$ initioQUANTUM ESPRESSO package [22], following the simulation setup used in our previous work [4], but with some parameters adjusted. We have used a projector augmented wave (PAW) [23,24] pseudopotential and a Perdew-Burke-Ernzerhof generalized gradient approximation (PBEGGA) [25] for the exchangecorrelation functional, with a cutoff energy of $80 \mathrm{Ry}$ for the plane-wave expansion and $500 \mathrm{Ry}$ for the charge density. The integration in reciprocal space is conducted over a uniformly spaced Monkhorst-Pack grid [26] of $14 \times 18 \times 1$ points for $1 \times 1$ zigzag, and $14 \times 16 \times 1$ points for $1 \times 1$ armchair. The resolution of this grid in the $x$ direction is reduced as the period increases, up to $2 \times 18 \times 1$ points for $10 \times 10$ zigzag, and $2 \times 16 \times 1$ points for $10 \times 10$ armchair. Convergence is achieved when changes in total energy between consecutive self-consistent steps are less than $1 \times 10^{-8} \mathrm{Ry}$ and all forces are smaller than $1 \times 10^{-4} \mathrm{Ry} /$ a.u. After this relaxation, the unit cells are replicated in the $x$ and $y$ directions to generate the superlattices of alternated zigzag [Fig. 1(c)] or armchair [Fig. 1(d)] layers of graphene and boron nitride. Table I collects the equilibrium lattice vectors $a_{\alpha}$, number of replicated unit cells $N_{\alpha}$, and simulation lengths $L_{\alpha}$. Further relaxation is
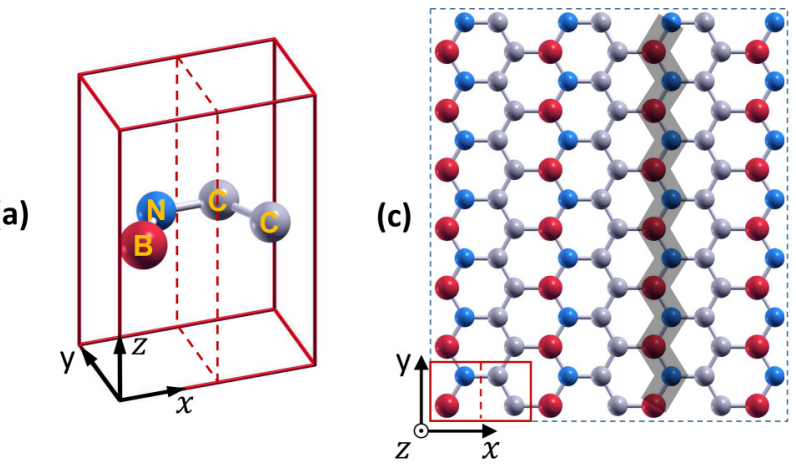

(b)

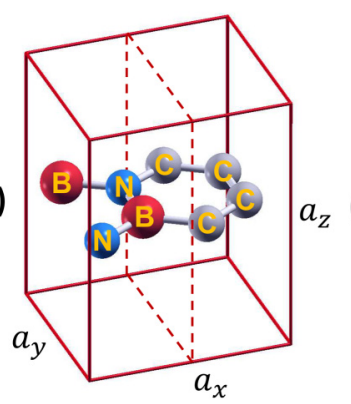

(d)

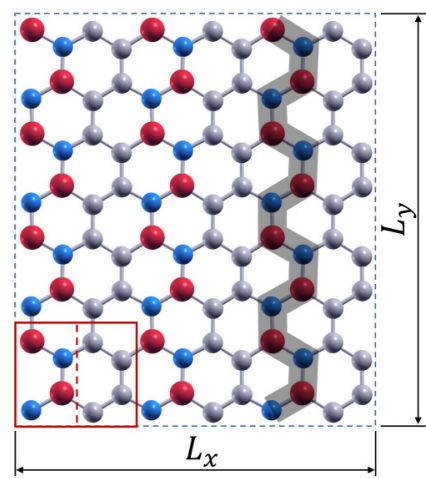

FIG. 1. Superlattice unit cells for (a) the $1 \times 1$ zigzag interface, and (b) the $1 \times 1$ armchair interface. Atomic structures of the $1 \times 1$ superlattices for $(c)$ the zigzag interface, and (d) the armchair interface.

conducted with the interatomic potential within both HLD and MD simulations.

In this work, the ultimate goal is to calculate the thermal conductivity of these superlattices using their phonon properties. To this end, we have implemented a mode-dependent equation from kinetic theory [27], to obtain the phonon thermal conductivity in the $\alpha$ th direction as

$$
K_{\alpha}(k, v)=\sum_{k, v} C_{p h}(k, v) v_{\alpha}^{2}(k, v) \tau(k, v),
$$

where $C_{p h}(k, v)$ is the heat capacity, $v_{\alpha}(k, v)$ is the $\alpha$ th component of the group velocity, and $\tau(k, v)$ is the phonon lifetime or relaxation time. We have employed Bose-Einstein statistics to estimate the heat capacities and HLD simulations to predict the group velocities. The relaxation times are extracted from MD simulations using a frequency domain normal mode analysis. The MD simulations naturally include fourth- and higher-order intrinsic scattering processes and the extrinsic effects of boundaries and interfaces [28]. Our approach contrasts with other methods such as the standard single-mode relaxation time approximation [29] and the iterative solution to the Boltzmann transport equation [30], where only third-order scattering processes are considered and extrinsic effects are only empirically included.

The phonon properties are functions of the polarization branches $v$ and the wave vectors $k$. These wave vectors are specified within the first Brillouin zone (BZ), which is a rectangular prism with reciprocal lattice vectors $b_{\alpha}$ given by $2 \pi / a_{\alpha}$. Based on the number of replicated unit cells $N_{\alpha}$, the 
TABLE I. Number of atoms and size of the MD simulations domains $\left(L_{x} \times L_{y}\right)$, number of replicated unit cells in the $\mathrm{x}\left(N_{x}\right)$ and $\mathrm{y}$ $\left(N_{y}\right)$ directions, number of atoms in the unit cells, and equilibrium lattice parameters $a_{x}$ and $a_{y}$ for each superlattice period and interface configuration.

\begin{tabular}{|c|c|c|c|c|c|c|c|c|c|c|c|c|}
\hline \multirow[b]{2}{*}{ Period } & \multicolumn{6}{|c|}{ Zigzag } & \multicolumn{6}{|c|}{ Armchair } \\
\hline & Atoms & $\begin{array}{c}L_{x} \times L_{y} \\
(\mathrm{~nm} \times \mathrm{nm})\end{array}$ & $N_{x} \times N_{y}$ & $\begin{array}{c}\text { Atoms } \\
\text { (unit cell) }\end{array}$ & $\begin{array}{c}a_{x} \\
(\mathrm{~nm})\end{array}$ & $\begin{array}{c}a_{y} \\
(\mathrm{~nm})\end{array}$ & Atoms & $\begin{array}{c}L_{x} \times L_{y} \\
(\mathrm{~nm} \times \mathrm{nm})\end{array}$ & $N_{x} \times N_{y}$ & $\begin{array}{c}\text { Atoms } \\
\text { (unit cell) }\end{array}$ & $\begin{array}{c}a_{x} \\
(\mathrm{~nm})\end{array}$ & $\begin{array}{c}a_{y} \\
(\mathrm{~nm})\end{array}$ \\
\hline $1 \times 1$ & 14720 & $20.04 \times 19.86$ & $46 \times 80$ & 4 & 0.4357 & 0.2482 & 14720 & $20.00 \times 19.87$ & $40 \times 46$ & 8 & 0.5001 & 0.4319 \\
\hline $2 \times 2$ & 15360 & $20.84 \times 19.86$ & $24 \times 80$ & 8 & 0.8686 & 0.2482 & 14720 & $19.99 \times 19.82$ & $20 \times 46$ & 16 & 0.9993 & 0.4309 \\
\hline $3 \times 3$ & 15360 & $20.81 \times 19.86$ & $16 \times 80$ & 12 & 1.3007 & 0.2483 & 15456 & $20.96 \times 19.83$ & $14 \times 46$ & 24 & 1.4971 & 0.4310 \\
\hline $4 \times 4$ & 15360 & $20.79 \times 19.86$ & $12 \times 80$ & 16 & 1.7326 & 0.2483 & 14720 & $19.95 \times 19.82$ & $10 \times 46$ & 32 & 1.9945 & 0.4309 \\
\hline $5 \times 5$ & 16000 & $21.64 \times 19.87$ & $10 \times 80$ & 20 & 2.1642 & 0.2484 & 14720 & $19.95 \times 19.82$ & $8 \times 46$ & 40 & 2.4932 & 0.4308 \\
\hline $7 \times 7$ & 13440 & $18.16 \times 19.88$ & $6 \times 80$ & 28 & 3.0268 & 0.2485 & 15456 & $20.93 \times 19.82$ & $6 \times 46$ & 56 & 3.4886 & 0.4308 \\
\hline $10 \times 10$ & 12800 & $17.28 \times 19.88$ & $4 \times 80$ & 40 & 4.3204 & 0.2485 & 14720 & $19.92 \times 19.81$ & $4 \times 46$ & 80 & 4.9798 & 0.4307 \\
\hline
\end{tabular}

wave vectors are specified as $k=b_{\alpha}\left(n_{\alpha} / N_{\alpha}\right)$, where $n_{\alpha}$ is an integer with allowed values in the range of $-N_{\alpha} / 2$ and $N_{\alpha} / 2$. We have imposed the symmetry of the BZ by computing the phonon properties only for the irreducible wave vectors in the first quadrant of this zone, that is, $0 \leqslant n_{x} \leqslant N_{x} / 2$ and $0 \leqslant n_{y} \leqslant N_{y} / 2$. The thermal conductivities are estimated at room temperature $(\mathrm{T}=300 \mathrm{~K})$, well below the classic limit in graphene $(\sim 2100 \mathrm{~K})$ [31]. Therefore, we have incorporated quantum effects by using Bose-Einstein statistics to estimate the heat capacities $C_{p h}(k, v)$ as functions of the phonon frequencies $\omega(k, v)$ as

$$
C_{p h}(k, v)=\frac{k_{B} x(k, v)^{2} e^{x(k, v)}}{\left(e^{x(k, v)}-1\right)^{2}},
$$

where $k_{\mathrm{B}}$ is the Boltzmann constant, and $x(k, v)=$ $\hbar \omega(k, v) / k_{B} T$, with $\hbar$ being the reduced Planck's constant. In previous works $[4,32]$, we found that the heat capacities are practically unaffected by the superlattice period. Consequently, we focus here on the variation of group velocities and relaxation times.

The group velocities have been predicted using harmonic lattice dynamics (HLD) simulations with the General Utility Lattice Program (GULP) [33] and a Tersoff-type [34] interatomic potential developed by Kinac1 et al. [21]. Initially, the unit cell structures were further relaxed with the interatomic potential in GULP using a Broyden-Fletcher-Goldfarb-Shanno (BFGS) algorithm. The HLD simulations are then performed to solve the eigenvalue problem for the atoms in the unit cells, to obtain the normal mode eigenvectors and the harmonic phonon frequencies $\omega(k, v)$. The eigenvectors are used to precisely locate the acoustic phonon modes, those in which all atoms move in phase [35]. The frequencies are used to build the dispersion curves and then to obtain the group velocities by calculating the slope of the curves with central differences. In addition, the HLD curves for the superlattices have been validated with dispersion curves from DFPT simulations. This validation was necessary because the dispersion curves were not included in the original work where the parametrization of the potential was developed [21]. We have built the DFPT curves with interatomic force constants previously obtained from dynamical matrices calculated for a uniform wave-vector grid of $16 \times 16 \times 1 k$ points [4].
The phonon lifetimes are computed using a NMD approach applied to a set of molecular dynamics (MD) trajectories $[3,36]$. The MD trajectories are generated at room temperature with the LAMMPS package [37]. The simulation domains are rectangular prisms with widths $L_{x}$ and lengths $L_{y}$ of approximately $20 \mathrm{~nm}$ and a fixed depth $L_{z}$ of $1.5 \mathrm{~nm}$ that contain around 15000 atoms. The depth is sufficiently long to preclude self-interaction of atoms in the $z$ direction as periodic boundary conditions are imposed in all directions of the simulation cell. Once the simulation cell is built, the superlattices are first relaxed at a constant temperature of $300 \mathrm{~K}$ and pressure of 0 bars for $1200 \mathrm{ps}$ with a time step of $0.2 \mathrm{fs}$. The superlattices are next equilibrated at constant volume and a temperature of $300 \mathrm{~K}$ for $800 \mathrm{ps}$. The equations of motion are then integrated at constant volume and energy for 419 ps ( $2^{21}$ steps). In this final integration, the atomic velocities are stored to disk in windows of $105 \mathrm{ps}\left(2^{19}\right.$ steps $)$ every $6.4 \times 10^{-3} \mathrm{ps}\left(2^{5}\right.$ steps $)$. Once the simulations are completed, the atomic velocities, equilibrium atomic positions, and phonon mode eigenvectors are used to calculate the time derivative of the normal mode coordinates $\dot{q}(k, v, t)$ [35]. We apply the Fourier transform to the autocorrelation of this time derivative to generate the phonon power spectrum $T(k, v, \omega)$ as [36]

$$
T(k, v, \omega)=\lim _{\tau_{0} \rightarrow \infty} \frac{1}{2 \tau_{0}}\left|\frac{1}{\sqrt{2} \pi} \int_{0}^{\tau_{0}} \dot{q}(k, v, t) e^{-i \omega t} d t\right|^{2},
$$

where $\tau_{0}$ is the time of the simulation sampling window equal to $105 \mathrm{ps}$. This value is one order of magnitude longer than the longest relaxation time predicted here $(\sim 15 \mathrm{ps})$. The power spectrum of an individual phonon at wave vector $k$ and polarization $v$ is averaged over results from four sampling windows and five MD simulations with different initial conditions. The phonon lifetimes are finally predicted by fitting the averaged power spectra to the Lorentzian function,

$$
T(k, v, \omega) \approx \frac{I(k, v)}{\left[\frac{\omega_{A}(k, v)-\omega}{\Gamma(k, v)}\right]^{2}+1},
$$

where the fitting parameters are the intensity of the peak $I(k, v)$, the anharmonic angular phonon frequency at the center of the peak $\omega_{A}(k, v)$, and the half width at half maximum of the peak $\Gamma(k, v)$. The phonon relaxation time $\tau(k, v)$ is calculated as the inverse of the full width at half maximum, $2 \Gamma(k, v)$. Equation (4) is fitted to the discrete points yielded 
by Eq. (3). This fit is made with an iteratively reweighted least-squares (IRLS) method with a Cauchy weight function [38]. The fit's precision is very sensitive to the initial guess for $\Gamma(k, v)$, which has to be carefully chosen at an arbitrary frequency. We have automated the process by conducting the fitting in two steps. In the first step, the intensity $I(k, v)$ is set equal to the maximum energy of the phonon spectrum, and the angular frequency $\omega_{A}(k, v)$ is set equal to the frequency at the maximum energy. Then, the data are fitted to Eq. (4) with $\Gamma(k, v)$ as the only adjusted parameter. The initial guesses for $\Gamma(k, v)$ are $0.04 \mathrm{rad} / \mathrm{ps}$ for $f \leqslant 5 \mathrm{THz}, 0.06 \mathrm{rad} / \mathrm{ps}$ for $5 \mathrm{THz}<f \leqslant 40 \mathrm{THz}$, and $0.1 \mathrm{rad} / \mathrm{ps}$ for $f>40 \mathrm{THz}$. In the second step, three parameters are adjusted and the outputs from the first step are taken as the initial guesses.

\section{RESULTS AND DISCUSSION}

\section{A. Phonon dispersion curves}

In this section we present the phonon dispersion curves as functions of wave vectors along the $k$-space directions $\left[\begin{array}{lll}0 & 1 & 0\end{array}\right]$ and $\left[\begin{array}{lll}1 & 0 & 0\end{array}\right]$ for the superlattices $1 \times 1$ zigzag (Fig. 2) and $1 \times 1$ armchair (Fig. 3). The density of states (DOS) is plotted beside the dispersion curves. The dispersion curves exhibit 12 polarization branches for the zigzag interface and 24 branches for the armchair interface, in correspondence with the number of degree of freedoms in the zigzag unit cell $(4 \times 3=12)$ and armchair unit cell $(8 \times 3=24)$. All solid lines are obtained from HLD simulations. The optical (O) modes are shown in black, out-of-plane acoustic (ZA) in blue, transversal acoustic (TA) in green, and longitudinal acoustic

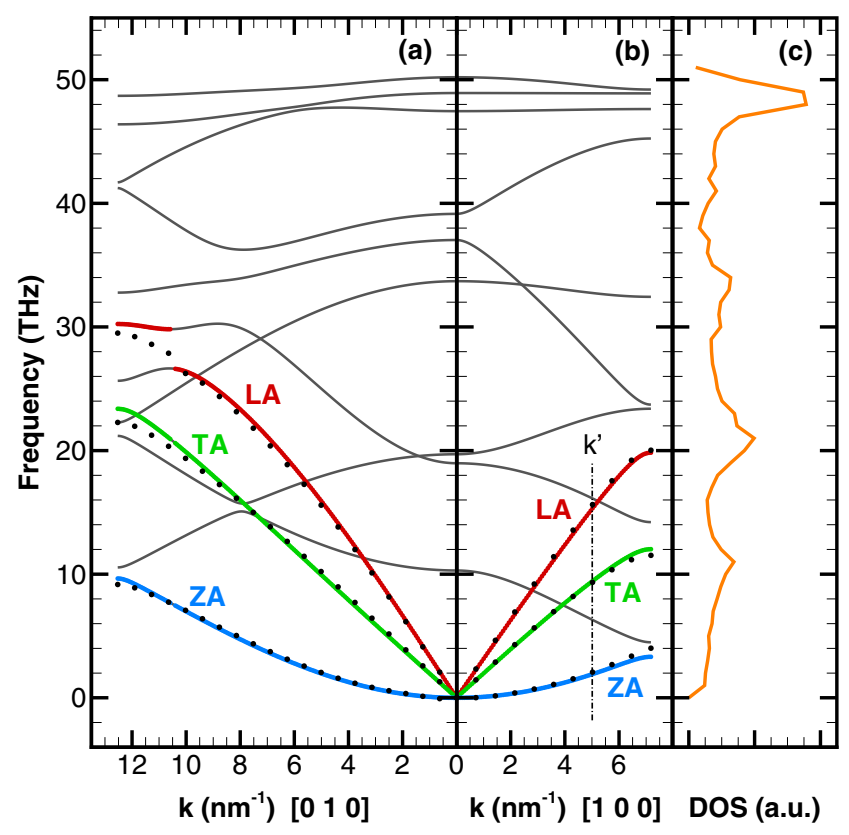

FIG. 2. Dispersion curves for the $1 \times 1$ zigzag superlattice (a) along the $k$-space direction [ $\left[\begin{array}{ll}0 & 1\end{array}\right]$ ], and (b) along the $k$-space direction $\left[\begin{array}{lll}1 & 0 & 0\end{array}\right]$. (c) Phonon density of states. The solid lines represent data from the HLD simulations. The black solid lines represent the optical modes, and the highlighted lines represent the acoustic branches. The dots represent data from the DFPT simulations.

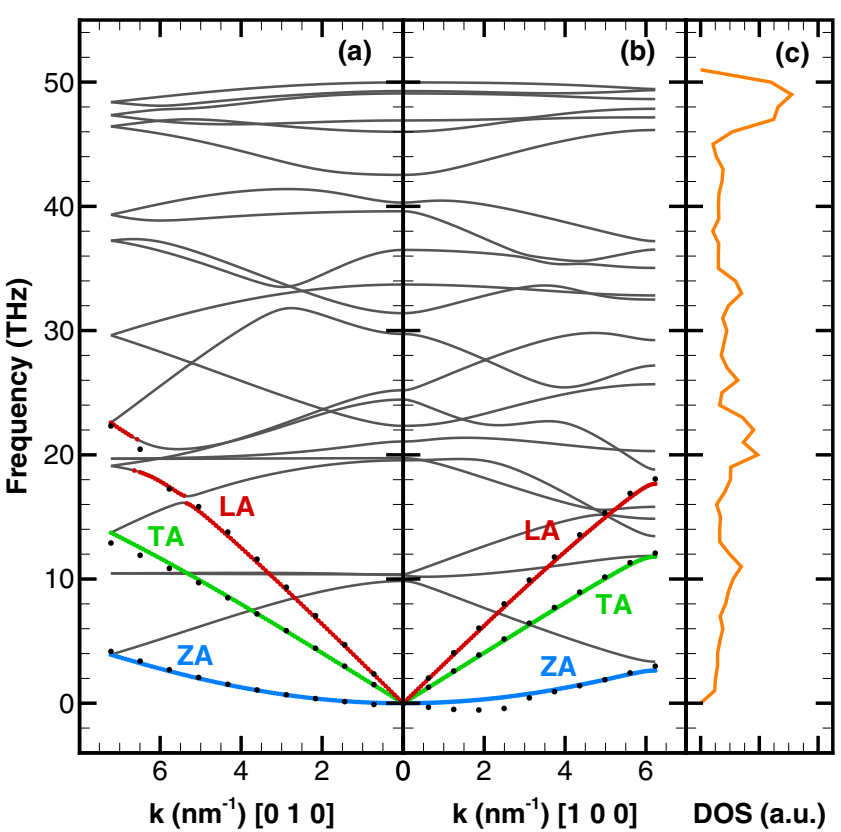

FIG. 3. Dispersion curves for the $1 \times 1$ armchair superlattice (a) along the $k$-space direction [0 10 ], and (b) along the $k$ - space direction $\left[\begin{array}{lll}1 & 0 & 0\end{array}\right]$. (c) Phonon density of states. The solid lines represent data from the HLD simulations. The black solid lines represent the optical modes, and the highlighted lines represent the acoustic branches. The dots represent data from the DFPT simulations.

(LA) in red. For clarity, the results from DFPT simulations (dots) are shown only for the acoustic branches. For the $1 \times 1$ zigzag superlattice, these branches are responsible for $70.7 \%$ of the thermal transport in the $y$ direction and $57.6 \%$ in the $x$ direction. There is excellent agreement between HLD and DFPT results, confirming that the Tersoff parametrization used in the HLD simulations reproduces the main features of the dispersion curves of these short-period superlattices. In our previous work [4], we showed that this agreement extends to higher-frequency optical branches, especially those with higher slopes. We emphasize that these DFPT results are shown here only for validation purposes. The group velocities are estimated from the HLD curves to be consistent with the lifetime estimation approach, which uses the same Tersoff potential [21].

The superlattice structure disrupts the continuity of the acoustic branches in the [ $\left[\begin{array}{lll}0 & 1 & 0\end{array}\right]$ direction, as illustrated by the segmented branches highlighted in Figs. 2(a) and 3(a) for the TA and LA modes. This disruption has also been observed in the in-plane phonon dispersion of vertically stacked superlattices [39], caused by the spatial confinement of phonons. We have verified that the eigendisplacements of phonons in these segments are consistent with the behavior of acoustic modes. Overall, the segmented branches resemble the behavior of the acoustic phonons in pure graphene [40] and boron nitride [41]. The most distinct feature of these dispersion curves lies on the frequencies reached by the acoustic branches in each direction, which is determined by the configuration of the unit cell. The unit cell of the $1 \times 1$ armchair superlattice is approximately squared, causing the acoustic branches to reach 
similar frequencies (up to $20 \mathrm{THz}$ ) in both directions. However, the unit cell for the $1 \times 1$ zigzag superlattice is rectangular, with $a_{x}$ approximately twice $a_{y}$, causing the acoustic branches

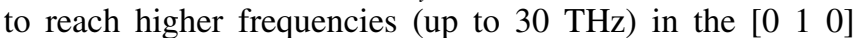
direction than in the $\left[\begin{array}{lll}1 & 0 & 0\end{array}\right]$ direction. These differences are responsible for the anisotropic behavior of the thermal conductivity in these superlattices. The frequencies reached by the acoustic branches are reflected on the phonon DOS. The DOS of the $1 \times 1$ zigzag superlattice exhibits its highest peak at $48 \mathrm{THz}$, in agreement with the DOS of graphene and boron nitride [4,12]. The second-highest peak emerges at $20 \mathrm{THz}$, and the third-highest peak at $10 \mathrm{THz}$. These lower-intensity peaks appear around the maximum frequencies reached by the LA and TA modes in the $\left[\begin{array}{lll}1 & 0 & 0\end{array}\right]$ direction. This pattern is also found in the DOS of graphene and boron nitride [4], but with the peaks shifted according to the maximum frequencies of the acoustic modes: 8 and $18 \mathrm{THz}$ for boron nitride and 14 and $26 \mathrm{THz}$ for graphene. The DOS of superlattices with greater periods (not shown) exhibit a similar shape, but with a greater concentration at $48 \mathrm{THz}$ and a more evenly distributed DOS at lower frequencies, without an energy gap in the phonon dispersion.

\section{B. Phonon group velocities}

In this section we discuss the effect of the superlattice period on the group velocities of individual acoustic modes and average group velocities. Figure 4 shows the acoustic branches for zigzag superlattices with periods 1, 2, and 5, plotted alongside the acoustic branches of graphene and boron nitride. The length of the first BZ in the [ $\left[\begin{array}{lll}1 & 0 & 0\end{array}\right]$ direction decreases as the period increases, as indicated by the vertical dotted line for the $2 \times 2$ superlattice, and the vertical dashed line for the $5 \times 5$ superlattice. The length of the first BZ in the $\left[\begin{array}{lll}0 & 1 & 0\end{array}\right]$ direction

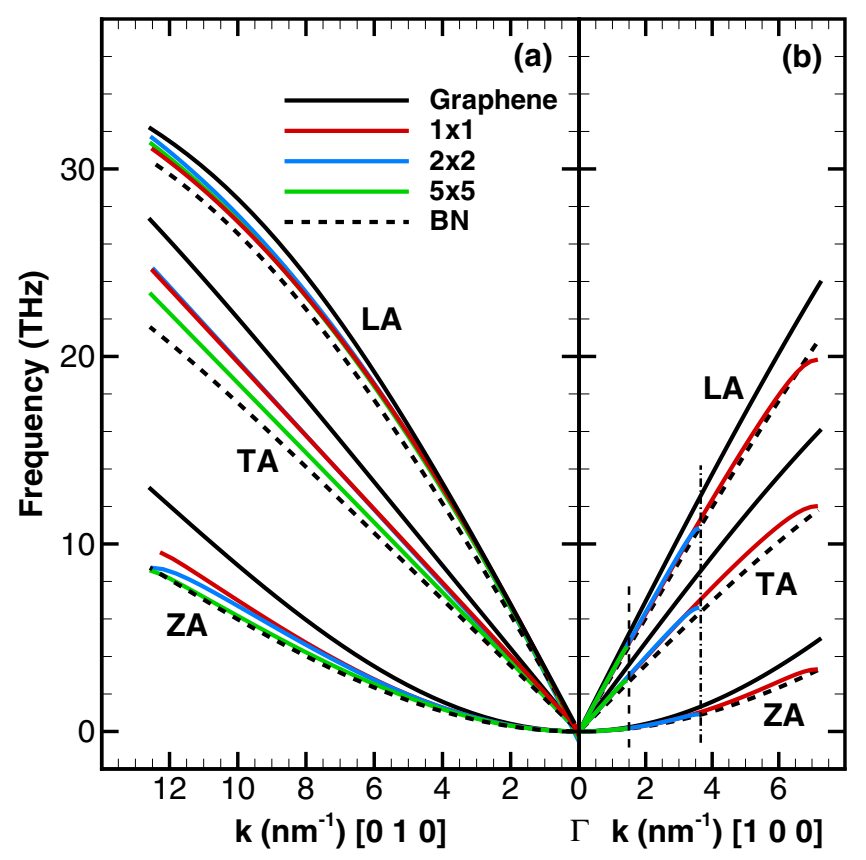

FIG. 4. Acoustic dispersion curves (a) along the $k$-space direction $\left[\begin{array}{lll}0 & 1 & 0\end{array}\right]$, and (b) along the $k$-space direction $\left[\begin{array}{lll}1 & 0 & 0\end{array}\right]$. remains unchanged because the lattice vector in this direction is the same for all periods. For the sake of comparing the acoustic branches of the superlattices with those of graphene and boron nitride, the segmented curves in the [0 $\left.\begin{array}{lll}0 & 1 & 0\end{array}\right]$ direction are adjusted in Fig. 4(a) to fit the linear dispersion of TA and LA modes at low frequency. In all cases, the curves corresponding to the superlattices are in between those of graphene and boron nitride. The TA modes in both directions exhibit the greatest differences in slope (group velocities) between graphene and boron nitride. The group velocities of the TA modes in graphene $(14.91 \mathrm{~nm} / \mathrm{ps})$ are approximately 34\% higher than those in boron nitride $(11.12 \mathrm{~nm} / \mathrm{ps})$. This difference is reduced to $15 \%$ for the LA modes. All the ZA branches show a parabolic dispersion with approximately zero group velocity at the gamma point. In the $\left[\begin{array}{lll}1 & 0 & 0\end{array}\right]$ direction, the acoustic branches are truncated at smaller frequencies as the period increases; however, their group velocities are preserved and they have approximately the same value as those for boron nitride. In the $\left[\begin{array}{lll}0 & 1 & 0\end{array}\right]$ direction, the group velocities for the $1 \times 1$ and $2 \times 2$ superlattices are closer to the average group velocities of graphene and boron nitride, and they decrease as the period increases.

We now address the variation of the average squared group velocity with the phonon frequency and superlattice period. Figure 5 shows this variation in the $x$ and $y$ directions for the zigzag superlattices. The squared group velocities are predominantly higher at period 1 in both directions. At this period, the greatest averages are observed at $12 \mathrm{THz}$ in the $x$ direction $\left(50 \mathrm{~nm}^{2} / \mathrm{ps}^{2}\right)$ and $14 \mathrm{THz}$ in the $y$ direction $\left(75 \mathrm{~nm}^{2} / \mathrm{ps}^{2}\right)$. These peaks represent the frequency level at which the interplay between acoustic and optical branches maximizes the average group velocities, which occurs at wave vectors around the center of the irreducible Brillouin zone $\left(k / k_{\max }=0.5\right)$ in both directions, as observed in Fig. 2. On the other hand, the wells at $10 \mathrm{THz}$ account for the combined effect of steep acoustic branches at the center of the zone and flat optical branches at the borders. In the $x$ direction, the

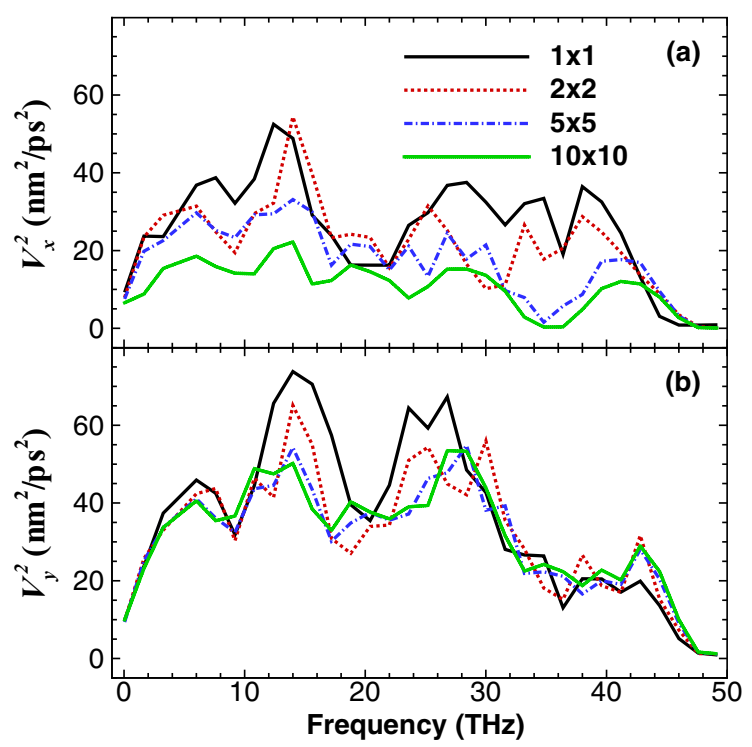

FIG. 5. Squared phonon group velocities for the zigzag superlattices in (a) the $\mathrm{x}$ direction, and (b) the $y$ direction. 


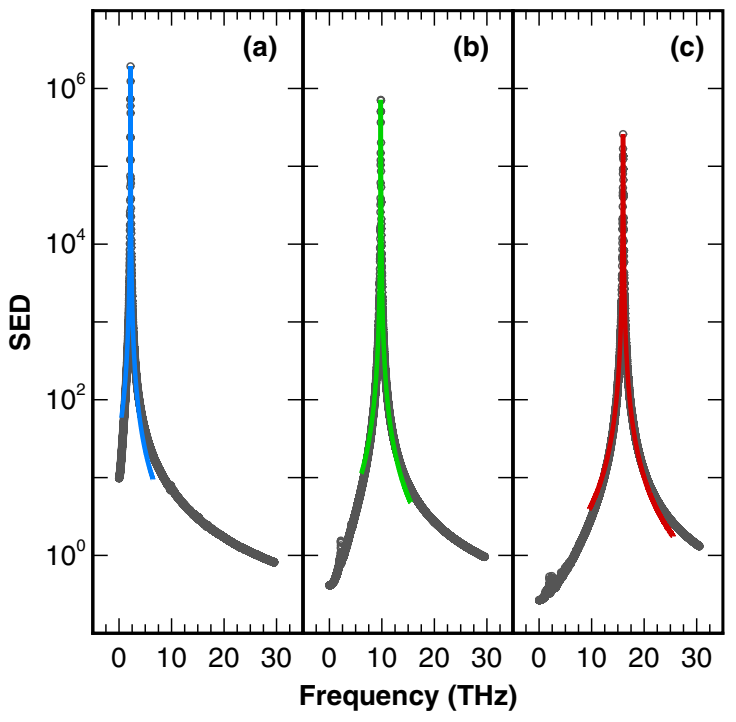

FIG. 6. Phonon power spectra for the $1 \times 1$ zigzag superlattice at wave vector $k^{\prime}=\left[17 \pi / 23 a_{x}, 0,0\right]$ for the acoustic polarizations (a) out-of-plane ZA, (b) transverse TA, and (c) longitudinal LA.

velocities are consistently reduced as the period increases. The average velocity in the frequency range between 4 and $20 \mathrm{THz}$ drops from 33.08 to $29.61 \mathrm{~nm}^{2} / \mathrm{ps}^{2}$ from period 1 to period 2 , a decreasing ratio that is more than twice higher than that from period 2 to period $5\left(1.30 \mathrm{~nm}^{2} / \mathrm{ps}^{2} /\right.$ period). In the $y$ direction, the average velocity in this frequency range drops from 48.74 to $40.21 \mathrm{~nm}^{2} / \mathrm{ps}^{2}$ from period 1 to period 2 , and then remains practically unchanged for higher periods.

\section{Phonon lifetimes}

In this section we describe the behavior of phonon lifetimes in both the superlattices and equivalent samples of graphene and boron nitride. We start by presenting a sample of the fitted power spectra. Figure 6 shows the discrete power spectra (circles) and fitting curves (solid lines) as functions of the anharmonic phonon frequencies $(f=\omega / 2 \pi)$ for three isolated peaks, which correspond to the acoustic polarizations at the wave vector $k^{\prime}=\left[17 \pi / 23 a_{x}, 0,0\right]$ for the $1 \times 1$ zigzag superlattice [shown in Fig. 2(b)]. By incorporating the harmonic eigendisplacements in the spectral analysis, we estimate the lifetimes from fully isolated peaks. We obtain peaks without any signature from other frequencies, meaning that our harmonic eigendisplacements are an excellent description of the anharmonic modes at room temperature. This mode-bymode analysis allows us to fit the spectra considering all data points within five orders of magnitude below the point with the maximum energy. This range of data minimizes the root mean squared error (RMSE), whose averaged value for the $1 \times 1$ zigzag superlattice (353) is fairly low compared to the range of energy values $\left(10^{0}-10^{6}\right)$. The uncertainty due to fitting is estimated with a cross-validation approach by varying the range of the data points used for the fitting, considering data points within three to five orders of magnitude of the maximum data value. The average uncertainty for all phonon lifetimes increases from $2.75 \%$ at period 1 to $4.62 \%$ at period 10 . The peak intensity is the greatest for the ZA mode and decreases

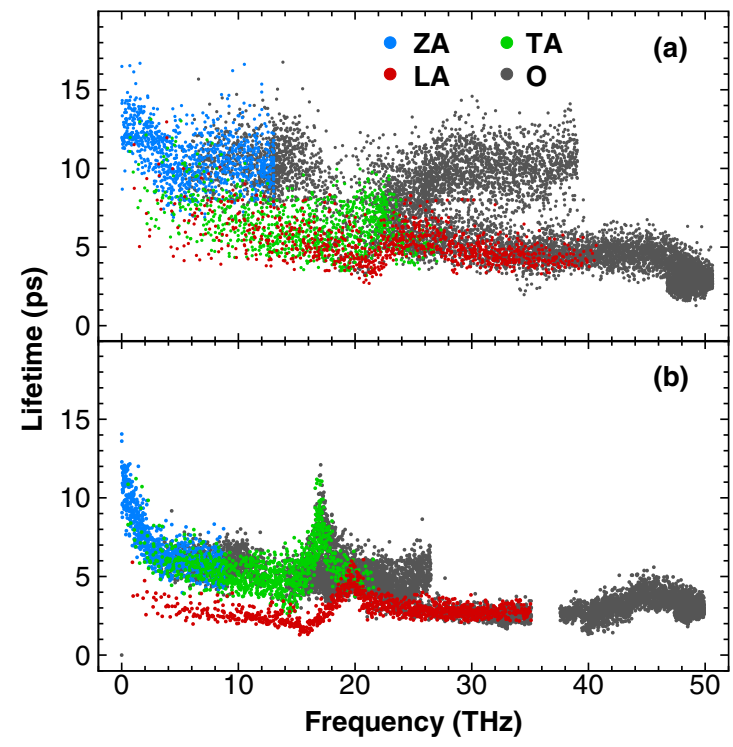

FIG. 7. Phonon lifetimes for (a) graphene, and (b) boron nitride.

for higher-frequency modes (TA and LA), and so does the phonon lifetime. This trend is replicated at all wave vectors. In addition, the anharmonic frequencies for these acoustic modes are fairly close to the corresponding harmonic frequencies, only shifted by $2.83 \%$ for the ZA mode, $1.13 \%$ for the TA mode, and $1.12 \%$ for the LA mode. The average shift in frequency is below $2 \%$ for all superlattice periods; therefore, it is reasonable to calculate the group velocities from HLD simulations.

We now present in Fig. 7 the phonon lifetime distributions for graphene and boron nitride. These lifetimes are calculated for a simulation domain with the same size and four-atom unit cell structure used for the $1 \times 1$ zigzag superlattice. Thus, direct comparisons can be made between the lifetimes for this superlattice structure and the lifetimes for the bulk materials. Despite the similarities in atomic masses and lattice constants between graphene and boron nitride, the magnitude and spectral dependence of their lifetimes are substantially different. The lifetimes of acoustic modes in graphene exhibit a weak dependence on frequency, in qualitative agreement with previous works [12,42]. However, we show here that they are distributed within shorter ranges. The lifetimes of the ZA modes are in the range of $8-16 \mathrm{ps}$ up to $14 \mathrm{THz}$, and the lifetimes of the TA and LA modes are in the range of 4-10 ps up to $26 \mathrm{THz}$. In contrast, Qiu and Ruan [42] obtained lifetimes for the ZA modes in the range of 10-40 ps, and Feng et al. [12] in the range of 10-30 ps. We attribute these discrepancies to differences in the resolution of the power spectra and the sensitivity of the fitting procedure, especially of those peaks with the highest intensities at low frequencies. The lifetimes of acoustic modes in boron nitride show a stronger dependence on frequency $\left(\sim \omega^{-0.2}\right)$, but still in disagreement with the expected $\omega^{-2}$ scaling at low frequency [43]. The absence of a $\omega^{-2}$ scaling in these samples of graphene and boron nitride indicates that the phonon-phonon scattering (intrinsic effect) is not dominant at low frequencies, as it is the case for bulk argon [44] and silicon [45]. The verification of this scaling in 2D materials will require much bigger simulation domains; 


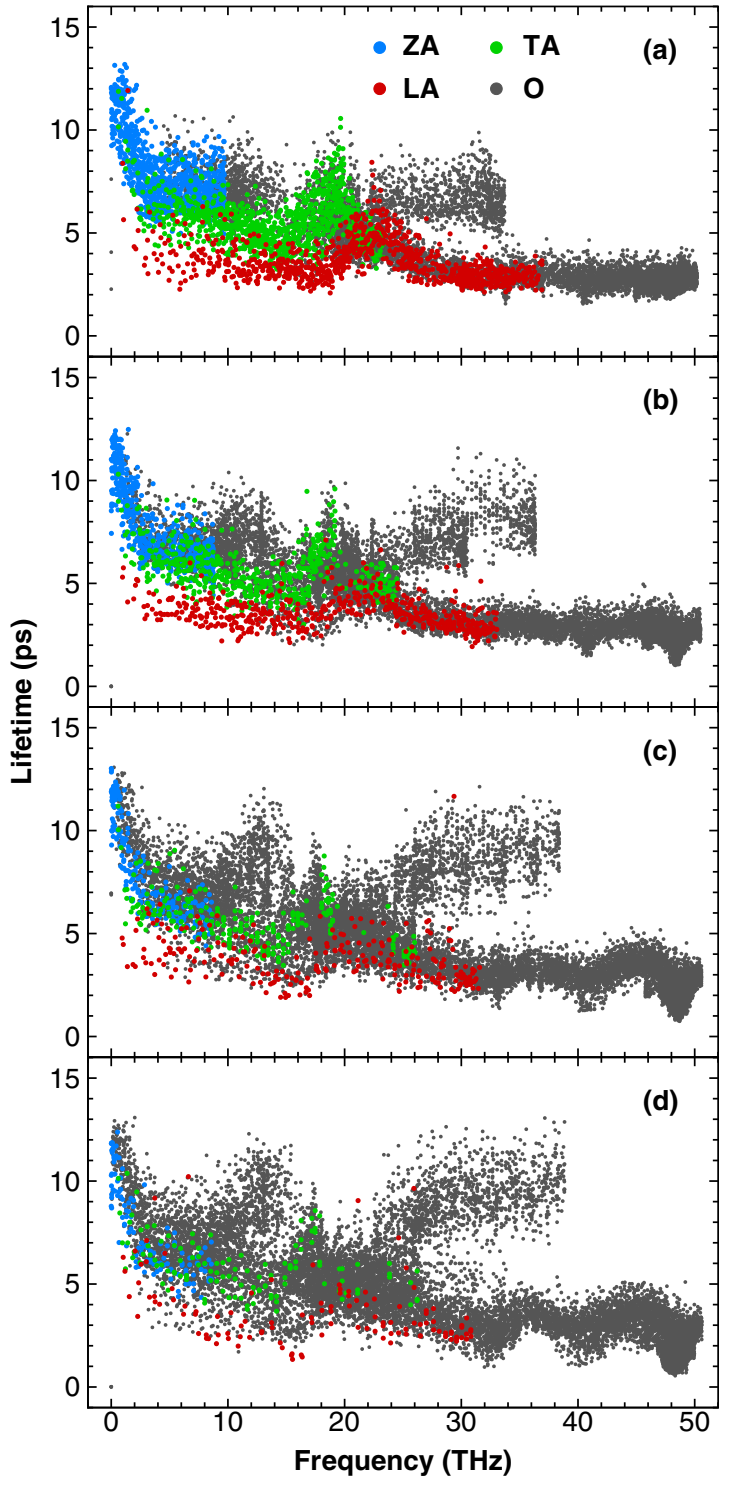

FIG. 8. Phonon lifetimes for the superlattices (a) $1 \times 1$ zigzag, (b) $2 \times 2$ zigzag, (c) $5 \times 5$ zigzag, and (d) $10 \times 10$ zigzag.

nonetheless, going beyond the size adopted in this work becomes computationally prohibitive for spectral analyses. In the frequency range between 4 and $16 \mathrm{THz}$, the average lifetime of $\mathrm{ZA}, \mathrm{TA}$, and $\mathrm{O}$ modes in boron nitride $(\sim 6 \mathrm{ps})$ is approximately half of that in graphene $(\sim 16 \mathrm{ps})$. In this same range, we also note the smaller lifetimes of the LA modes $(\sim 3 \mathrm{ps})$ in boron nitride compared to those of the TA modes $(\sim 6 \mathrm{ps})$. At intermediate frequencies $(16-22 \mathrm{THz})$, the lifetime distribution in boron nitride develops a peak at $16 \mathrm{THz}$ for the TA and $\mathrm{O}$ modes, and at $20 \mathrm{THz}$ for the LA mode, coinciding with the location of the second peak in the phonon DOS $(\sim 18 \mathrm{THz})$.

The lifetime distributions for the zigzag superlattices are shown in Fig. 8 for periods 1, 2, 5, and 10. The dimensions of the simulation cell are kept approximately constant for all periods $(\sim 20 \mathrm{~nm} \times 20 \mathrm{~nm})$, such that lifetimes are not affected by the size of the samples and the effect of the period can be addressed independently. The lifetimes for the $1 \times 1$ superlattice resemble the scaling observed in boron nitride, but with average values in between those of graphene and boron nitride. The resolution of the acoustic modes decreases at higher periods because the number of replicated unit cells is smaller. At the same time, there are more optical modes due to branch folding; therefore, the whole resolution of the BZ remains approximately constant. Overall, lifetimes show a weak dependence on the superlattice period, indicating that the phonon transport is dominated by coherent effects, i.e., long-wavelength phonons traveling across the interfaces without scattering [17]. However, it is noticeable that the maximum lifetimes of optical modes in the frequency range of $10-20 \mathrm{THz}$ and $24-40 \mathrm{THz}$ increase from approximately 9 ps at period 1 to 12 ps at period 10. Phonons experiencing this increase come from flat optical branches at intermediate frequencies in the phonon dispersion, with very small group velocities and short wavelengths. Thus, these phonons are expected to scatter diffusively at the interfaces [17]. This increase is not as significant for flat optical branches at higher frequencies $(40-50 \mathrm{THz})$ due to the much higher phonon DOS.

\section{Thermal conductivities}

The thermal conductivities are predicted from the phonon properties discussed in previous sections and presented in Fig. 9 as functions of the superlattice period for both directions and interface configurations. The uncertainties due to the statistical error (not shown) are less than $1 \%$ in all cases, estimated by systematically removing one MD simulation from the average power spectra. Overall, these thermal conductivities follow the same trend found in our previous work [4], but with absolute values that are approximately $25 \%$ greater. This increase responds to the improved fitting procedure of the peaks and, most importantly, the smaller $\Delta \omega$ implemented here $(0.06 \mathrm{rad} / \mathrm{ps})$, resulting in power spectra with twice the resolution. This higher resolution reveals interesting physics in the relaxation time distribution by capturing peaks with higher

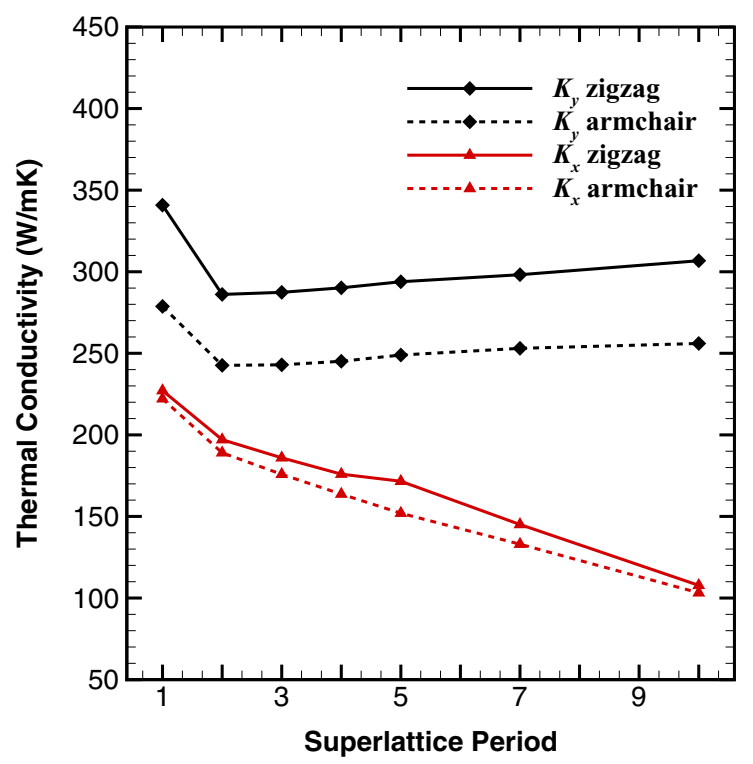

FIG. 9. Variation of the thermal conductivity with the superlattice period and interface structure. 
intensities. We first focus on the highest thermal conductivities, predicted at period 1 for the zigzag configuration. At this period, the thermal conductivity in the $x$ direction $(227.15 \mathrm{~W} / \mathrm{mK})$ is approximately $15 \%$ smaller than that of a same-size sample of boron nitride $(266.43 \mathrm{~W} / \mathrm{mK})$, and $60 \%$ smaller than that of graphene $(564.61 \mathrm{~W} / \mathrm{mK})$. The thermal conductivity in the $y$ direction $(340.82 \mathrm{~W} / \mathrm{mK})$ is closer to the average conductivity of graphene and boron nitride. These results contrast with those found by Gard et al. [20] in 3D superlattices of silicon and germanium with perfect interfaces, where the thermal conductivities at the shortest period were found to be higher than those of the constituent materials. The authors showed how the significant difference in the atomic masses of silicon (28.09 amu) and germanium (72.64 amu) induces an energy gap in the phonon dispersion, causing a substantial increase of the relaxation times at this limit. The formation of such a gap in our superlattices is precluded due to the similarities between the masses of carbon (12.01 amu), boron (10.81 amu), and nitrogen (14.01 amu) atoms.

The response of the thermal conductivities to an increase in the periodicity follows the same trend in both interface configurations. The conductivities for the zigzag superlattices are always higher than those for the armchair superlattices, on average $7 \%$ higher in the $x$ direction and $19 \%$ in the $y$ direction. Note that the greatest decrease in the conductivities occurs between periods 1 and 2, approximately a $13 \%$ decrease in the $x$ direction and $16 \%$ in the $y$ direction. In the $x$ direction, the thermal conductivities monotonically decrease with increasing periodicity, which is consistent with a phonon transport regime dominated by coherent effects [17]. The rate of decrease from period 1 to $2(\sim 30 \mathrm{~W} / \mathrm{mK} /$ period $)$ is approximately three times higher than that from period 2 to $10(\sim 11 \mathrm{~W} / \mathrm{mK} /$ period $)$. This monotonic decrease up to period $10\left(a_{x}=4.32 \mathrm{~nm}\right)$ is in qualitative agreement with results from nonequilibrium molecular dynamics (NEMD) simulations [19], where coherent effects were found dominant for period lengths smaller than $6 \mathrm{~nm}$, regardless of the sample size. In the $y$ direction, the thermal conductivities first decrease from period 1 to $2(\sim 50 \mathrm{~W} / \mathrm{mK} /$ period $)$, and then they slightly recover for periods higher than $2(\sim 2 \mathrm{~W} / \mathrm{mK} /$ period $)$. This recovery indicates that diffusive effects are dominant in the $y$ direction; i.e., the thermal conductivity increases with increasing periodicity because there are fewer sites for the diffuse scattering of short-wavelength phonons at the interfaces. In a previous work [32], we estimated the thermal conductivities of these superlattices using MD simulations with the Green-Kubo (GK) method. These simulations also predicted the greatest decrease in the conductivities from period 1 to 2 . For some periods, the results presented here are in good agreement with these GK results, e.g., in the $y$ direction for the zigzag superlattice at period $1(369.4 \pm 66.2 \mathrm{~W} / \mathrm{mK})$ and period 2 $(296.0 \pm 49.9 \mathrm{~W} / \mathrm{mK} \mathrm{W} / \mathrm{mK})$. However, direct comparisons with these results have to be made with particular caution, especially considering the significant high statistical uncertainties associated with the GK simulations of these superlattices.

Figure 10 shows the contribution of each phonon frequency to the thermal conductivity for the zigzag and armchair superlattices with periods $1,2,5$, and 10 . The total thermal conductivity is proportional to the areas under these curves. For both interface configurations, $90 \%$ of the contributions

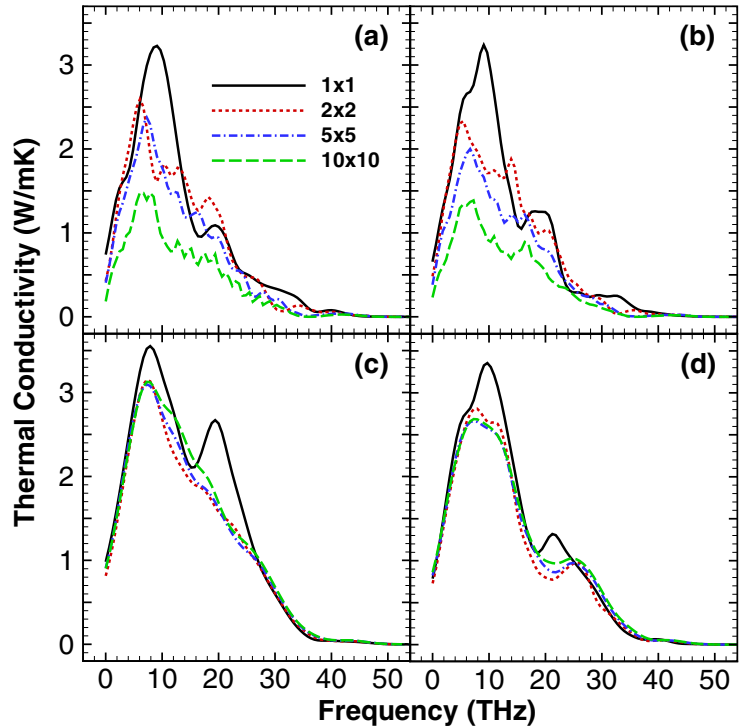

FIG. 10. Thermal conductivity contributions as functions of the phonon frequencies for the superlattices (a) zigzag in the $x$ direction, (b) armchair in the $x$ direction, (c) zigzag in the $y$ direction, and (d) armchair in the $y$ direction.

come from phonon frequencies up to approximately $23 \mathrm{THz}$ in the $x$ direction and $26 \mathrm{THz}$ in the $y$ direction, regardless of the period. In all cases, the contributions at period 1 significantly deviate from those at larger periods. This deviation extends over the frequency range between 6 and $14 \mathrm{THz}$ in the $x$ direction, and between 6 and $26 \mathrm{THz}$ in the $y$ direction. For periods higher than 2 , the contributions within these ranges are consistently reduced in the $x$ direction, and remain practically unchanged in the $y$ direction. These findings are in direct correlation with the group velocity variations shown in Fig. 5, denoting the more relevant role of these phonon properties in the anisotropic behavior of the thermal transport. Interestingly, the maximum peaks in the contributions at period 1 emerge at approximately $10 \mathrm{THz}$, coinciding with the location of the first well in the group velocities. However, at $10 \mathrm{THz}$ also emerges the first peak in the phonon DOS. Therefore, these maxima are driven by the higher concentration of states at this frequency. Similarly, the second peak in the contributions appears at approximately $20 \mathrm{THz}$ and is more significant in the $y$ direction for the $1 \times 1$ zigzag superlattice, coinciding with the location of the second well in the group velocities and the second peak in the phonon DOS. In Fig. 10(c), it is noteworthy the slight increase in the contributions for periods higher than 2 in the frequency range 10-20 THz, confirming the origin of the increase in the thermal conductivities in the $y$ direction, coming from the increase in the relaxation times reported within this range, as discussed in Sec. III 14;C. Finally, the contributions from frequencies higher than $26 \mathrm{THz}$ in all superlattices converge and ultimately decay to zero, due to the combined effect of the low group velocities, relaxation times, and specific heats of high-frequency phonons.

\section{CONCLUSIONS}

In this work, we have implemented a normal mode decomposition (NMD) approach to predict the phonon properties 
and thermal conductivities at $300 \mathrm{~K}$ of seven short-period superlattices of boron nitride and graphene, with zigzag and armchair interfaces. The simulations have been conducted on fully relaxed squared samples of these superlattices. We have provided a rigorous description of the dispersion curves and density of states of the superlattices, including a comparison between the acoustic branches in the superlattices with those in the constituent materials. We have found that $90 \%$ of the thermal conductivity is contributed from phonons with frequencies up to $23 \mathrm{THz}$ in the $x$ direction and $26 \mathrm{THz}$ in the $y$ direction, regardless of the period length. Our mode-by-mode analysis has revealed the dominant role of group velocities (coherent effects) in the $x$ direction, causing a monotonic decrease in the thermal conductivity as the period increases. Notably, the rate of this decrease from the first to the second period ( $\sim 30 \mathrm{~W} / \mathrm{mK} /$ period) is three times higher than that at greater periods $(\sim 11 \mathrm{~W} / \mathrm{mK} /$ period $)$. We have also detected the less relevant role of diffusive scattering (incoherent effects), particularly evident in the $y$ direction, where the increase in the relaxation times of short-wavelength phonons causes a slight increase in the thermal conductivities ( $\sim 2 \mathrm{~W} / \mathrm{mK} /$ period $)$.

\section{ACKNOWLEDGMENTS}

This work was supported by the Natural Sciences and Engineering Research Council of Canada (NSERC), through the Discovery and the Collaborative Research and Development (CRD) grant programs. Computations were performed on the GPC supercomputer at the SciNet HPC Consortium. SciNet is funded by the Canada Foundation for Innovation under the auspices of Compute Canada; the Government of Ontario; Ontario Research Fund-Research Excellence; and the University of Toronto. Parts of the computations were also made on the supercomputer Guillimin from McGill University, managed by Calcul Québec and Compute Canada. The operation of this supercomputer is funded by the Canada Foundation for Innovation (CFI), NanoQuébec, and RMGA and the Fonds de Recherche du Québec_-Nature et technologies (FRQ-NT).
[1] A. J. H. McGaughey, E. S. Landry, D. P. Sellan, and C. H. Amon, Appl. Phys. Lett. 99, 131904 (2011).

[2] C. Yu and G. Zhang, J. Appl. Phys. 113, 044306 (2013).

[3] S. C. Huberman, J. M. Larkin, A. J. H. McGaughey, and C. H. Amon, Phys. Rev. B 88, 155311 (2013).

[4] C. da Silva, F. Saiz, D. A. Romero, and C. H. Amon, in Proceedings of the ASME 2015 International Mechanical Engineering Congress and Exposition (IMECE) (ASME, Houston, 2015), Vol. 8A.

[5] X. Xu, L. Pereira, Y. Wang, J. Wu, K. Zhang, X. Zhao, S. Bae, C. Bui, R. Xie, J. Thong, B. Hong, K. Loh, D. Donadio, B. Li, and B. Özyilmaz, Nat. Commun. 5, 3689 (2014).

[6] Y. Lin and J. W. Connell, Nanoscale 4, 6908 (2012).

[7] X. Wei, Y. Wang, Y. Shen, G. Xie, H. Xiao, J. Zhong, and G. Zhang, Appl. Phys. Lett. 105, 103902 (2014).

[8] A. A. Balandin and D. L. Nika, Mater. Today 15, 266 (2012).

[9] R. Courtland, IEEE Spectrum 50, 14 (2013).

[10] C. da Silva, J. Sborz, D. A. Romero, and C. H. Amon, in Proceedings of the Canadian Society for Mechanical Engineering International Conference (CSME, Toronto, 2014).

[11] S. Chen, Q. Wu, C. Mishra, J. Kang, H. Zhang, K. Cho, W. Cai, A. A. Balandin, and R. S. Ruoff, Nat. Mater. 11, 203 (2012).

[12] T. Feng, X. Ruan, Z. Ye, and B. Cao, Phys. Rev. B 91, 224301 (2015).

[13] K. S. Novoselov, A. K. Geim, S. V. Morozov, D. Jiang, Y. Zhang, S. V. Dubonos, I. V. Grigorieva, and A. A. Firsov, Science 306, 666 (2004).

[14] A. K. Geim and I. V. Grigorieva, Nature 499, 419 (2013).

[15] Z. Liu, L. Ma, G. Shi, W. Zhou, Y. Gong, S. Lei, X. Yang, J. Zhang, J. Yu, K. P. Hackenberg, A. Babakhani, J. C. Idrobo, R. Vajtai, J. Lou, and P. M. Ajayan, Nat. Nanotechnol. 8, 119 (2013).

[16] Q. Sun, Y. Dai, Y. Ma, W. Wei, and B. Huang, RSC Adv. 5, 33037 (2015).

[17] M. Maldovan, Nat. Mater. 14, 667 (2015).

[18] J. Ravichandran, A. K. Yadav, R. Cheaito, P. B. Rossen, A. Soukiassian, S. J. Suresha, J. C. Duda, B. M. Foley, C.-H. Lee, Y. Zhu, A. W. Lichtenberger, J. E. Moore, D. a Muller, D. G.
Schlom, P. E. Hopkins, A. Majumdar, R. Ramesh, and M. A. Zurbuchen, Nat. Mater. 13, 168 (2014).

[19] T. Zhu and E. Ertekin, Phys. Rev. B 90, 195209 (2014).

[20] J. Garg, N. Bonini, and N. Marzari, Nano Lett. 11, 5135 (2011).

[21] A. Kınacı, J. B. Haskins, C. Sevik, and T. Çağın, Phys. Rev. B 86, 115410 (2012).

[22] P. Giannozzi, S. Baroni, N. Bonini, M. Calandra, R. Car, C. Cavazzoni, D. Ceresoli, G. L. Chiarotti, M. Cococcioni, I. Dabo, A. Dal Corso, S. de Gironcoli, S. Fabris, G. Fratesi, R. Gebauer, U. Gerstmann, C. Gougoussis, A. Kokalj, M. Lazzeri, L. MartinSamos et al., J. Phys. Condens. Matter 21, 395502 (2009).

[23] P. E. Blöchl, Phys. Rev. B 50, 17953 (1994).

[24] G. Kresse and D. Joubert, Phys. Rev. B 59, 1758 (1999).

[25] J. P. Perdew, K. Burke, and M. Ernzerhof, Phys. Rev. Lett. 77, 3865 (1996).

[26] J. D. Pack, and H. J. Monkhorst, Phys. Rev. B 16, 1748 (1977).

[27] J. M. Ziman, Electrons and Phonons (Oxford, New York, 2001).

[28] T. Feng and X. Ruan, J. Nanomater. 2014, 206370 (2014).

[29] D. L. Nika, E. P. Pokatilov, A. S. Askerov, and A. A. Balandin, Phys. Rev. B 79, 155413 (2009).

[30] L. Lindsay, W. Li, J. Carrete, N. Mingo, D. A. Broido, and T. L. Reinecke, Phys. Rev. B 89, 155426 (2014).

[31] E. Pop, V. Varshney, and A. K. Roy, MRS Bull. 37, 1273 (2012).

[32] F. Saiz, C. da Silva, and C. H. Amon, in Proceedings of the ASME 2015 International Mechanical Engineering Congress and Exposition (IMECE) (ASME, Houston, 2015), Vol. 8B.

[33] J. D. Gale and A. L. Rohl, Mol. Simul. 29, 291 (2003).

[34] J. Tersoff, Phys. Rev. B 37, 6991 (1988).

[35] M. T. Dove, Introduction to Lattice Dynamics (Cambridge University Press, Cambridge, 1993).

[36] J. M. Larking, J. E. Turney, A. D. Massicotte, C. H. Amon, and A. J. H. McGaughey, J. Comput. Theor. Nanosci. 11, 249 (2014).

[37] S. Plimpton, J. Comput. Phys. 117, 1 (1995).

[38] P. J. Huber, Robust Statistics (John Wiley \& Sons, Cambridge, 1981). 
[39] A. A. Balandin, E. P. Pokatilov, and D. L. Nika, J. Nanoelectron. Optoelectron. 2, 140 (2007).

[40] L. Lindsay and D. A. Broido, Phys. Rev. B 81, 205441 (2010).

[41] L. Lindsay and D. A. Broido, Phys. Rev. B 84, 155421 (2011). [42] B. Qiu and X. Ruan, Appl. Phys. Lett 100, 193101 (2012).
[43] J. Callaway, Phys. Rev. 113, 1046 (1959).

[44] J. E. Turney, J. A. Thomas, A. J. H. McGaughey, and C. H. Amon, in Proceedings of the ASME/JSME 2011 8th Thermal Engineering Joint Conference (ASME, Honolulu, 2011).

[45] A. S. Henry and G. Chen, J. Comput. Theor. Nanosci. 5, 141 (2008). 